\title{
Gerodontology: An Interdisciplinary Approach
}

\author{
Vikram Jha ${ }^{1}$, Anuya Hemant Patankarr ${ }^{2}$, Rameshwari Singhall ${ }^{1}$, Pavitra Rastogii ${ }^{1}$, Shuchi Tripathi ${ }^{1}$ \\ and Nandlal ${ }^{1}$ \\ ${ }^{1}$ Department of Periodontology, Faculty of Dental Sciences, KGMU, Lucknow, India \\ ${ }^{2}$ Department of Prosthodontics, MA Rangoonwala College of Dental Sciences and Research Centre, Pune, Maharashtra, India
}

${ }^{*}$ Corresponding author: Rameshwari Singhal, Associate Professor, Department of Periodontology, Faculty of Dental Sciences, KGMU, Lucknow, India, Tel: 0019134320107; E-mail: rsinghal23@hotmail.com

Received date: May 02, 2017; Accepted date: May 04, 2017; Published date: May 10, 2017

Copyright: @ 2017 Jha V, et al. This is an open-access article distributed under the terms of the Creative Commons Attribution License, which permits unrestricted use, distribution, and reproduction in any medium, provided the original author and source are credited.

Citation: Jha V, Patankar AH, Singhal R, et al. Gerodontology: An Interdisciplinary Approach. Periodon Prosthodon 2016, 3:1.

\section{Abstract}

The aim of this review is to throw light on the interrelationship between periodontics, restorative dentistry and prosthodontics in geriatric population. Increased life expectancy along with appropriate dental treatment in the younger ages leads to retention of more number of teeth in the advanced ages. To maintain these teeth disease free and in function in the older individuals is a multi-faceted challenge. This article describes the various restorative and prosthodontic treatment modalities that are commonly used in the dental rehabilitation of geriatric patients and their relationship with the periodontium and gingival health.

Keywords: Geriatric patients; Inter-relationship; Periodontics; Restorative dentistry; Prosthodontics

\section{Introduction}

With advances in medical sciences, longevity of age is on an increase. Life expectancy is expected to be around 80 years of age in India in 2050 as per data [1]. Longer the life span, longer is the time teeth are retained in the mouth. This coupled with preventive dental care that patients have received in their younger ages will lead to retention of a greater number of teeth in the geriatric patients.

Geriatric patients often face a multitude of medical ailments. Mostly, these occur in combination with each other. Some of the common ailments are diabetes mellitus, hypertension, myocardial infarction and stroke, osteoporosis, neurodegenerative disorders like Alzheimer's disease, Parkinsonism and dementia, respiratory ailments, osteoporosis and rheumatoid arthritis [2]. Often the physical and mental dexterity of the older individual is compromised, necessitating supportive care from care givers. In a report by Griffin et al, they have stated that there is a strong correlation between general and oral health in the elderly population [3]. The commonly affecting dental problems in the geriatric patients are caries (both coronal and radicular caries), periodontitis, tooth mobility, xerostomia (which most commonly occurs as a side effect of the drugs which are taken by the elderly patients), wear disorders i.e., attrition, abrasion and erosion, unreplaced missing teeth, broken or failing older dental restorations, mucosal diseases, oral cancer and alveolar ridge atrophy [4]. These problems ultimately lead to loss of teeth. Loss of teeth can be distressing and sometimes devastating as it can lead to serious psychosocial consequences which can affect the person's quality of life [5]. This manifestation is more pronounced in the elderly population.

Complete dental rehabilitation of geriatric patients involves a multidisciplinary approach. Along with enhancing and maintaining the health of the existing teeth, replacement of missing teeth is of utmost importance. Periodontics, restorative dentistry and prosthodontics are inseparable in this respect. A prosthesis can be termed as successful only if the abutment teeth and the surrounding periodontium are healthy. An improperly fabricated prosthesis may cause food entrapment and accumulation of plaque, eventually leading to progression of periodontal disease [6] Periodontal disease will lead to loss of attachment, tooth mobility and ultimately loss of tooth [7]. An increased rate of bone loss and periodontal breakdown was observed in patients over 70 years of age in a retrospective study by Papapanou et al. [8].

Prosthetic treatment options for partially dentate patients include removable prosthesis, fixed prosthesis and currently the most popular, implant prosthodontics. Proper diagnosis and treatment planning are of paramount importance in restoring function and esthetics for patients. This is of special significance in older patients, where the aim of the dentist should be restoration of dentition keeping in mind the various biologic, esthetic and functional parameters [9].

A smile is said to be esthetic only when both the pink and the white esthetics are in harmony. These two factors are inter-dependent on each other. It is critical that restorations are well contoured and well-polished, self-cleansing as well as esthetic in appearance. This will prevent food lodgement and accumulation of plaque. Not only will restorations fail in an 
unhealthy mouth but also improper restorations will lead to an unhealthy mouth. Andreana in his review article has rightly stated that it is essential for the clinician to understand the biologic foundation principles prior to restorative reconstructions of periodontally compromised teeth [10].

The aim of the review is to explain in detail the interdisciplinary relationship between periodontal therapy and prosthetic rehabilitation and between periodontal therapy and restorative dentistry in geriatric patients.

\section{Periodontal Therapy and Restorative Procedures}

The primary goal of restorative dentistry is restoration of form, function and esthetics while maintaining the biological, mechanical and esthetic principles. Advances in dental and material sciences have led to the availability of a variety of restorative materials which, in a restoration form, cannot be distinguished from a natural tooth. However, to fabricate such restorations which are in harmony with the gingiva and periodontal health is the primary challenge for most clinicians.

In older adults, dental caries especially root caries is rampant because of xerostomia. Root caries are more difficult to treat, due to difficulty in isolation. Studies have shown that root caries are more difficult to excavate due to their greater extent as compared to coronal caries [11] Also, accessibility is a problem, especially in molars 12 and the interproximal areas [13] where the prevalence of root caries is the highest. Restoration of the carious lesions is generally by the conventional filling methods. Chalmers has advocated the use of Minimal Intervention Dentistry for caries control in older individuals [14]. It focusses on early detection and prevention, remineralization; use of a range of restorations such as dental amalgams, composites or glass ionomer cements and surgical intervention only when required and only after disease has been controlled. Atraumatic restorative treatment (ART) is also indicated for older dentate institutionalised adults with a high caries rate. A study by da Mata et al has revealed that ART is as effective as conventional treatment for caries control [11]. Besides the Glass ionomer cements used act as fluoride releasing agents to limit caries progression.

Caries and periodontal disease affect the vitality of the pulp necessitating endodontic treatment. There is no contradiction to endodontic treatment as far as age is concerned [9]. In fact, whether a particular tooth needs to be retained in the oral cavity of an older adult depends on the prosthetic treatment plan. An endodontically treated tooth may serve as an abutment for a fixed partial denture, help in retention of a removable partial denture, support an attachment in a tooth supported overdenture or may help to maintain occlusal stability in cases of extensive wear [15]. Successful endodontic treatment may improve the prognosis of a periodontally compromised tooth, thus improving its longevity.

Age related changes also influence endodontic therapy. A study by Murray et al have documented decrease in the pulp repair capacity in geriatric patients after restorative treatments [16]. This along with increased calcification of the root canal system requires sound clinical judgement and skills on the part of the endodontist for a successful endodontic treatment. If all the principles of endodontics are followed along with good isolation and good technique, there is no reason for loss of the tooth in the aged patient.

\section{Periodontal Therapy and Prosthodontics}

This can be further subdivided into three categories

Periodontal therapy and Fixed Prosthodontics

Periodontal therapy and Removable Prosthodontics

Periodontal therapy and Implant Prosthodontics

\section{Periodontal Therapy and Fixed Prosthodontics}

The chief compliant of patients of the older age group is inability to eat satisfactorily due to missing teeth. They desire replacement using fixed prosthesis which includes implant supported crowns or crowns and bridges. In most cases, fixed prosthetic treatment options are preferred by the patient bearing in mind the physical health and the finances involved in rehabilitation using implants.

In tooth supported fixed prosthesis, it is critical to assess the abutment teeth via a periodontal perspective for long term and short term treatment planning. Tooth mobility, furcation involvement, severity of bone loss, bleeding/exudation from the gingival pockets are some of the periodontal factors that need to be examined prior to finalizing the prosthetic treatment plan [17]. It is essential to achieve periodontal health of the abutment teeth before proceeding with any further treatments. Similarly, one must bear in mind that the final restorations/ prosthesis that are to be cemented should be carefully planned and should be in harmony with the periodontium. The most important factor to be considered is the invasion of the biologic width by the margins of the fabricated restorations. This can result in gingival inflammation, loss of connective tissue attachment and bone loss. Surgical crown lengthening may be performed to maintain the biologic width and to maintain harmony between the periodontium and the prosthesis.

The design and fabrication of the fixed prosthesis should also be borne in mind to prevent periodontal breakdown and maintain gingival health. The older individuals have limited physical dexterity and are often dependent on care-givers for their necessities. The dentist and the dental technician should take care to fabricate prostheses which are self-cleansing and at the same time esthetic and functional. Open contacts between the crowns and the adjacent teeth/prosthesis should be avoided as chances of greater pocket depth and increased loss of clinical attachment levels were seen. This was confirmed in a study by Jernberg et al. [18]. The crowns should not be overcontoured and should be as smooth as 
possible with supragingival margins wherever possible. This will prevent food entrapment and thereby plaque accumulation.

Nowadays, even geriatric patients demand highly esthetic restorations for their anterior teeth. Often ceramic veneers and full ceramic crowns must be fabricated to fulfil the aesthetic demands of patients with deteriorated anterior dentitions. A study by Turk et al revealed no significant differences in the shear bond strength values when glass ceramics were adhesively luted to teeth of older adults as compared to the younger population [19].

Apart from tooth loss due to caries, another problem which plagues the older individuals is non-carious tooth loss, also known as tooth wear. It may be due to combination of attrition, erosion, abrasion and abfraction. The patient reports with inability to chew along with decreased lower facial height and an unesthetic appearance. Multidisciplinary treatment involving a prosthodontist, periodontist and restorative dentist is needed for complete rehabilitation of such a patient.

\section{Periodontal Therapy and Removable Prosthodontics}

Although from a periodontal point of view, fixed prosthodontic treatment is desirable, it is not always feasible in older adults due to financial and medical conditions especially in Kennedy's Class I and II situations. Removable prostheses are retained by means of clasps and retentive arms on the abutment teeth adjoining edentulous spaces. The faulty design of the prosthesis often causes food entrapment, which in turn increases plaque accumulation and chances of periodontitis. Studies by various authors have found high prevalence of gingivitis, plaque, and gingival recession on abutment teeth as compared to non-abutment teeth $[20,21]$. Increased interleukin-1beta levels in gingival crevicular fluid have been found by Kurtis et al in patients wearing removable partial dentures suggesting that such dentures are a risk factor for periodontal disease progression [22].

The design of the partial denture also plays a role in the progression of periodontal disease. In a study by Akaltan and Kaynak, lingual plates and lingual bars were used as major connectors in removable partial dentures [23]. Tooth mobility was significantly lesser in patients using lingual plates, due to the indirect retention provided by them. Similarly, other periodontal parameters did not show a significant difference between the two groups in patients maintaining adequate oral hygiene.

In a report published on the type of material to be used for partial dentures for patients suffereing from periodontal disease, the authors have preferred the use of acrylic plates over metal ones [24]. In periodontal patients, there may be additional tooth loss after fabrication of the prosthesis and hence a modification of the design may be necessary, which is easier in an acrylic plate as compared to a metal one.

\section{Periodontal Therapy and Implant Prosthodontics}

The primary criterion for successful implant therapy is osseointegration of the implant in a three dimensional prosthetically correct position. It has been proven that implant supported rehabilitations facilitate better quality of life and oral comfort and function [25]. This is especially true in older individuals where the primary complaint is inability to eat food properly due to missing teeth. Perel has rightly said that "Geriatric dentistry should begin with implant dentistry, so that implants are not used as merely a last resort" [26].

Even though age is not a contra-indication to implant treatment, it is essential to select a patient who can tolerate the surgical treatment [27]. The patient should also be mentally and physically healthy, and should have the mental and physical dexterity to be able to maintain adequate oral hygiene around the implants. It should also be possible for the patient to visit the dentist for recall appointments to prevent any biological and mechanical complications of the implant supported prosthesis [28].

Older adults are generally either partially or completely edentulous due to periodontal problems or tooth loss due to caries. Periodontitis propagates bone resorption, which over longer periods of edentulism results in limited vertical and horizontal residual ridge [29]. It has been previously documented in literature that both bone quality and quantity are affected by ageing [30]. There is decreased cancellous bone and increase in the porosity of the cortical bone. Ridge augmentation and guided bone regeneration procedures may be too invasive in older adults and the results are generally unsatisfactory [28]. A long term retrospective study by Maiorana and co-workers have identified old age as one of the risk factors which negatively influenced implant survival in cases where dental implants were placed in resorbed jaws augmented using appositional bone allografts [31]. Instead Schimmel et al have suggested the use of ultra-wide $6 \mathrm{~mm}$ implants in cases of severe ridge resorption. They also suggest the use of narrow diameter implants in horizontal ridge deficiencies, thus avoiding concomitant augmentation procedures in older individuals [28]. Implants are generally used in geriatric patients as an option to removable prosthesis, to support the existing removable prosthesis after loss of supporting abutment teeth, for stabilization of partial dentures and complete dentures with the help of various attachments.

A study by Petricevic et al have stated that implant supported fixed prosthesis were associated with better quality of life in older individuals as compared to tooth supported fixed prosthesis [25]. When comparing crestal bone loss around implants in younger and older patients, no significant differences were noted by Bryant and Zarb in their study [32]. Also, to prevent peri-implantitis, screw retained restorations must be preferred over cement retained ones to avoid remnants of excess cement in the soft tissues. 


\section{Conclusion}

Creation of a functional occlusion along with esthetics is the major aim of dental rehabilitation of geriatric patients. The periodontist, prosthodontist and the restorative dentist often have to work in harmony bearing this goal in mind. Healthy soft tissues will lead to success and survival of the dental restorations. The hard and the soft tissues of the mouth are a part of one stomatognathic system and are inter-dependent on each other.

\section{References}

1. Mithal A, Bansal B, Kyer CS, Ebeling P (2014) The Asia-Pacific Regional Audit-Epidemiology, Costs, and Burden of Osteoporosis in India 2013: A report of International Osteoporosis Foundation. Indian J Endocrinol Metab 18: 449-454.

2. Scannapieco FA, Cantos A (2016) Oral inflammation and infection, and chronic medical diseases: implications for the elderly. Periodontol 72: 153-175.

3. Griffin SO, Jones JA, Brunson D, Griffin PM, Bailey WD (2012) Burden of Oral Disease Among Older Adults and Implications for Public Health Priorities. Am J Public Health 102: 411-418.

4. Helgeson MJ, Smith BJ, Johnsen M, Ebert C (2002) Dental considerations for the frail elderly. Spec Care Dentist 22: 40-55.

5. Dable RA, Yashwante BJ, Marathe SS, Gaikwad BS, Patil PB, et al. (2014) Tooth loss--how emotional it is for the elderly in India? Oral Health Dent Manag 13: 305-310.

6. John P, Ambooken M, Kuriakose A, Mathew JJ (2005) The periorestorative interrelationship-expanding the horizons in esthetic dentistry. J Interdiscip Dentistry 5: 46-53.

7. Preshaw PM (2015) Detection and diagnosis of periodontal conditions amenable to prevention. BMC Oral Health 15: 5-10.

8. Papapanou PN, Wennström JL, Gröndahl K (1989) A 10-year retrospective study of periodontal disease progression. J Clin Periodontol 16: 403-411.

9. Nadig RR, Usha G, Kumar V, Rao R, Bugalia A (2011) Geriatric restorative care - the need, the demand and the challenges. J Conserv Dent 14: 208-214.

10. Andreana S (2010) Restorative options for the periodontal patient. Dent Clin North Am 54: 157-161.

11. DaMata C, Allen PF, McKenna G, Cronin M, O'Mahony D, et al. (2015) Two-year survival of ART restorations placed in elderly patients: A randomised controlled clinical trial. J Dent 43: 405-411.

12. Watanabe MG (2003) Root caries prevalence in a group of Brazilian adult dental patients. Braz Dent J 14: 153-156.

13. Louw AJ, Carstens IL, Hartshorne JE (1993) Root caries in a sample of elderly persons. J Dent Assoc S Afr 48: 183-187.

14. Chalmers JM (2006) Minimal intervention dentistry: part 2. Strategies for addressing restorative challenges in older patients. J Can Dent Assoc 72: 435-440.

15. Allen PF, Whitworth JM (2004) Endodontic considerations in the elderly. Gerodontology 21: 185-194.
16. Murray PE, Stanley HR, Matthews JB, Sloan AJ, Smith AJ (2002) Age-related odontometric changes of human teeth. Oral Surg Oral Med Oral Pathol Oral Radiol Endod 93: 474-482.

17. Hsu YT, Huang NC, Wang HL (2015) Relationship Between Periodontics and Prosthodontics: The Two-Way Street. Journal of Prosthodontics and Implantology 4: 4-11.

18. Jernberg GR, Bakdash MB, Keenan KM (1983) Relationship between proximal tooth open contacts and periodontal disease. J Periodontol 54: 529-533.

19. Türk AG, Cal E, Unal S, Güneri P, Ulusoy M, et al. (2017) Glassceramics bonding in geriatric patients: comparison with young teeth. Gerodontology 34: 49-56.

20. Yeung AL, Lo EC, Chow TW, Clark RK (2000) Oral health status of patients 56 years after placement of cobaltchromium removable partial dentures. J Oral Rehabil 27: 183-189.

21. do Amaral BA, Barreto AO, Gomes Seabra E, Roncalli AG, da Fonte Porto Carreiro A, et al. (2010) A clinical followup study of the periodontal conditions of RPD abutment and nonabutment teeth. J Oral Rehabil 37: 545-552.

22. Kurtiş B, Tüter G, Korkmaz T, Yücel A, Serdar M, et al. (2003) Clinical examination and interleukin-1beta levels in gingival crevicular fluid in patients treated with removable partial dentures. Int J Prosthodont 16: 59-63.

23. Akaltan F, Kaynak D (2005) An evaluation of the effects of two distal extension removable partial denture designs on tooth stabilization and periodontal health. J Oral Rehabil 32: 823-829.

24. Metal versus Acrylic Partial Removable Dentures for Patients with Periodontal Disease: A Review of the Clinical Effectiveness and Guidelines [Internet] (2016) Ottawa (ON): Canadian Agency for Drugs and Technologies in Health.

25. Petricevic N, Celebic A, Rener-Sitar K (2012) A 3-year longitudinal study of quality-of-life outcomes of elderly patients with implant- and tooth-supported fixed partial dentures in posterior dental regions. Gerodontology 29: 956-963.

26. Perel ML (2007) Implants and the elderly. Implant Dent 16: 225.

27. Grant BT, Kraut RA (2007) Dental implants in geriatric patients: a retrospective study of 47 cases. Implant Dent 16: 362-368.

28. Schimmel M, Müller F, Suter V, Buser D (2017) Implants for elderly patients. Periodontol 73: 228-240.

29. Carranza FA (2002) Bone Loss and Patterns of Bone Destruction. Carranza's Clinical Periodontology. 9th edn. Philadelphia, USA.

30. Ikebe K, Wada M, Kagawa R, Maeda Y (2009) Is old age a risk factor for dental implants?. Japanese Dental Science Review 45: 59-64.

31. Maiorana C, Poli PP, Borgonovo AE, Rancitelli D, Frigo AC, et al. (2016) Long-Term Retrospective Evaluation of Dental Implants Placed in Resorbed Jaws Reconstructed With Appositional FreshFrozen Bone Allografts. Implant Dent 25: 400-408.

32. Bryant SR, Zarb GA (2003) Crestal bone loss proximal to oral implants in older and younger adults. J Prosthet Dent 89: 589-597. 